\title{
Field emission for resonance sensing in MEMS/NEMS
}

\author{
C.K. Yang ${ }^{1 *}$, A.J. le Fèbre ${ }^{2}$, G.. Pandraud ${ }^{3}$, E. van der Drift ${ }^{3}$, P.J. French ${ }^{1}$
}

${ }^{1}$ Electronic Instrumentation Labs, TU Delft, 2628CD, the Netherlands

${ }^{2} \mathrm{MESA}+$ Institute for Nanotechnology, University of Twente, 7522NM, the Netherlands

${ }^{3}$ Kavli Institute, TU Delft, 2628CJ, the Netherlands

${ }^{*}$ Corresponding author: c.k.yang@tudelft.nl

In the past decades, there is a considerable interest in the sensor community to move from micron to nano-devices, typically scaling of resonators such as cantilever beams. The scaled beams give advantages in faster response and higher sensitivity; however the detection of their resonance becomes challenging as dimensions scale down. In our work, we demonstrate the use of field emission characteristics as a detection method for scaled resonators. The advantages of using field emission are several: it is geometrically scalable without loss of signal, it has a high bandwidth and it can be integrated using standard fabrication processes.

Field emission sensing has been studied before in pressure sensors [1], data storage distance control [2] and RF MEMS switches [3]. Based on similar principles, we use the exponential relation of the emission current to the electric field $E$, which is a function of distance $E=\gamma(V / d)$ (where $\gamma$ is the field enhancement factor, $V$ the bias voltage and $d$ the electrode-tip distance), to sense the amplitude displacement of the cantilevers, and further extract the resonance information. Therefore, the displacement of a vibrating cantilever at its resonance frequency can be transduced into electrical current signals via field emission.

To understand the relationship between field emission and distance, we fabricated silicon tips with tip radius of 10 25 nm on fixed substrates (see Fig.1) and coated them with $6 \mathrm{~nm} \mathrm{Cr}$ and $40 \mathrm{~nm} \mathrm{Au}$. The measurements are carried out in an AFM system under UHV condition, the testing setup is the same as in [4]. The AFM system together with the fixed silicon tip allows precise control of the electrode-tip distances, ruling out the effect of cantilever bending of typical AFM probes. The measurement are carried out in two ways: in the first one we fix the bias voltage, and slowly vary the tip distance with a calibrated piezo stage; in the second, we change the bias voltage, measuring the displacement of the piezo stage while maintaining a constant emission current by using feedback to the tip position. Fig. 2 shows the measurement of emission current vs. relative sample-tip distance, under different fixed bias voltages. The measurement shows not only the exponential-like relationship of the emission current and the distance, but also the sensitivity change under different bias voltage and initial distances. Fig. 3 shows the measurement of bias voltage vs. relative sample-tip distance, at a fixed emission current of $3 \mathrm{nA}$. The bias vs. distance shows a linear relationship, the sensitivity of such detection method is extrapolated to be as high as $0.5 \mathrm{~V} / \mathrm{nm}$.

Finally, to measure the resonance detection by field emission, we use a commercial highly doped silicon AFM probe as the emitter. The AFM probe is actuated by piezo, and its tip is biased at $80 \mathrm{~V}$. The probe is held $\sim 100 \mathrm{~nm}$ away from the electrode in resonance and the resonance curve was obtained (see Fig. 4). The field emission current induced by the cantilever's mechanical resonance is fed to a low-noise current amplifier and measured by a spectrum analyzer. The measurement shows a resonance at $162.2 \mathrm{kHz}$ with a quality factor of $\sim 8000$, which corresponds to the specification of the AFM probe.

This work shows measurement of the field emission to distance relation. The relation is used to extract the resonant frequency information of the cantilever by transducing the displacement into electrical signal. The method may have wide applications in MEMS and especially NEMS device, due to its high sensitivity and its scaling effect independence. 


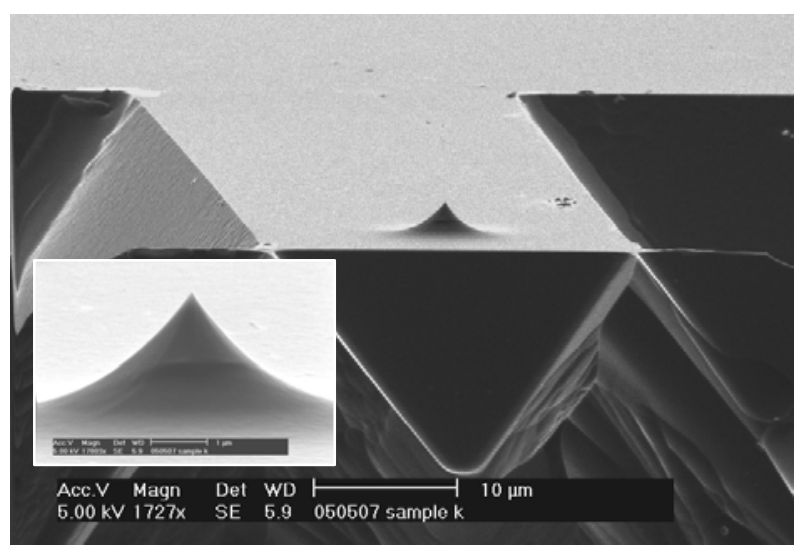

Fig.1. SEM image of silicon tip on fixed AFM substrate, tip radius is typically $10 \sim 25 \mathrm{~nm}$.
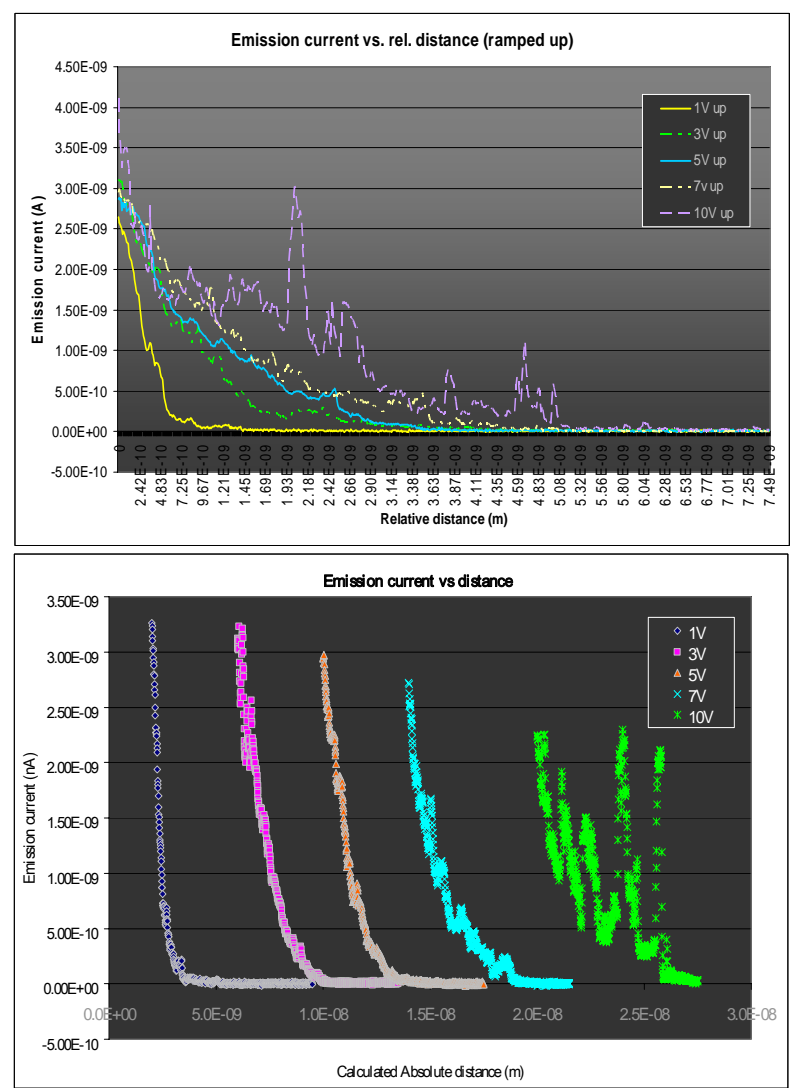

Fig. 2. Field emission current vs. relative tip-anode distance (2a), and vs. calculated absolute tip-anode distance (2b). Emission current measured under different biasing.

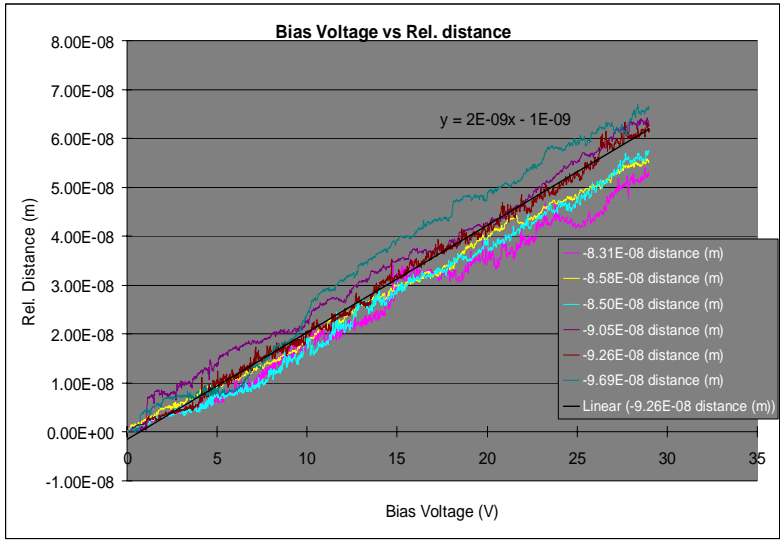

Fig. 3. Field emission voltage bias vs. relative tip-anode distance. Bias voltage is ramped up and down 3 times each. Distance in the inset represents initial tip-anode gap. First 3 are ramping up of the bias and later 3 are ramping down.

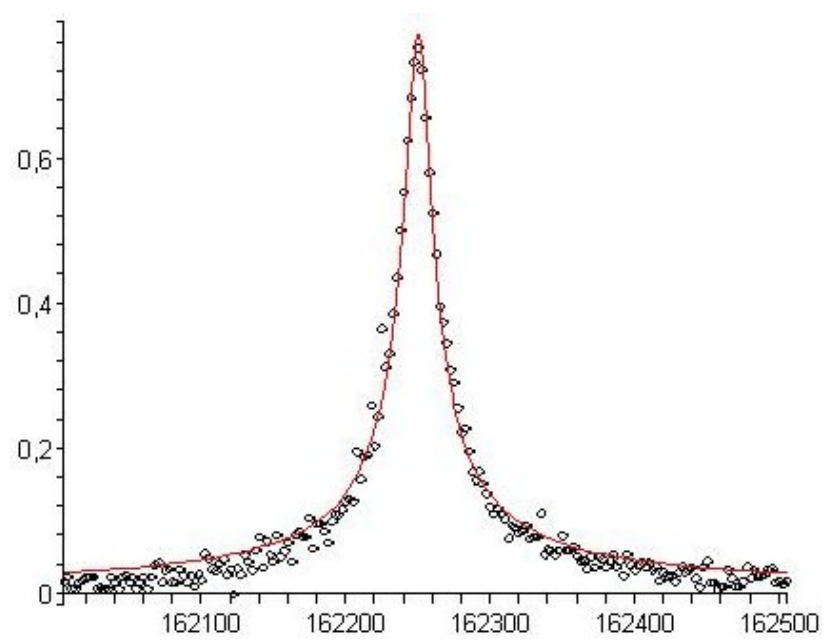

Fig. 4. AFM probe cantilever resonance curve obtained by field emission sensing. Resonance at $162.2 \mathrm{kHz}$ and quality factor of $\sim 8000$. The unit on the $\mathrm{x}$-axis is $\mathrm{Hz}$ and the $\mathrm{y}$-axis is arbitrary.

[1] H.H. Busta et al., J. Micromech. Microeng. 3, 49 (1993).

[2] le Febre, A.J. et al, J. Phys.: Conf. Ser. 61, 673, (2007)..

[3] K. Kakushima et al., J. Vac. Sci. Technol B, 24(2), 927 (2006).

[4] A.J. le Fèbre et al., accepted in the Proceeding of IVNC 2007. 